\title{
Analysis of the Structure and Expression of the Augmenter of Liver Regeneration (ALR) Gene
}

\author{
Roberto Giorda,* Michio Hagiya, ${ }^{\dagger}$ Tatsuya Seki, $^{\dagger}$ \\ Manabu Shimonishi, ${ }^{\dagger}$ Harumi Sakai, ${ }^{+}$James Michaelson, $^{\neq}$ \\ Antonio Francavilla,\$ Thomas E. Starzl, \$ and Massimo Trucco* \\ *Division of Immunogenetics, Department of Pediatrics, and \\ §Transplantation Institute, University of Pittsburgh, School of \\ Medicine, Pittsburgh, Pennsylvania, U.S.A. \\ †Pharmaceuticals Research Center, Toyobo Company, Limited, Ohtsu, \\ Shiga, Japan \\ ${ }^{\ddagger}$ Department of Pathology, Harvard Medical School, Boston, \\ Massachusetts, U.S.A.
}

\begin{abstract}
Background: The gene encoding the hepatotrophic factor Augmenter of Liver Regeneration (ALR) has recently been cloned in the rat. The availability of the mouse form of ALR would allow the analysis of the role of this factor in the physiology of liver and other organs, while the identification of the human homolog would allow the transfer of the great wealth of information that has been generated in animal models to clinically oriented pilot trials, and eventually the therapeutic application of this information.

Materials and Methods: Standard molecular biology approaches have been used to determine the genomic structure of the ALR gene in the mouse, and to characterize the ALR transcript and its protein product. The human ALR CDNA was also isolated and the amino acid sequence of the human gene product deduced. The mapping of mouse and human ALR genes on mouse and human chromosomes was then completed.
\end{abstract}

Results: The protein coding portion of the mouse ALR gene is comprised of three exons, the first containing the $5^{\prime}$ untranslated sequence and the initial 18 bases after the ATG translation initiation codon, the second exon encompasses 198 bases, and the third exon contains the remaining portion of the protein coding sequence. Rat, mouse, and human ALR genes (and protein products) were found to be highly conserved and preferentially expressed in the testis and in the liver. The ALR gene maps to the mouse chromosome 17 , in a region syntenic with human chromosome 16 , where the $T / t$ region has also been mapped.

Conclusions: ALR appears to be a protein with important physiologic properties, not exclusively limited to liver regeneration, with roles that are involved in the synthesis or stability of the nuclear and mitochondrial transcripts that are present in actively regenerating cells, particularly the germ cells of the testes.

\section{INTRODUCTION}

The extraordinary regenerative capacity of mammalian liver has long motivated investigations of factors involved in this aspect of multicellularity. One of the latest hepatotrophic factors described, named augmenter of liver regeneration (ALR),

Address correspondence and reprint requests to: Massimo Trucco, Children's Hospital of Pittsburgh, Rangos Research Center, 3705 Fifth Avenue at DeSoto Street, Pittsburgh, PA 15213, U.S.A. was purified from the cytosol of weanling rat livers (1). This factor, originally called hepatic regenerative stimulation substance (HSS), augments the cell proliferative response following hepatectomy in rats (2) and dogs (3).

The search for ALR began in 1931, when McJunkin and Breuhaus (4) reported that the modest regeneration following a limited (30$45 \%$ ) hepatectomy in rats was amplified by an intraperitoneal postoperative injection of rat 
liver homogenate. This regeneration augmenting effect was shown by Teir and Ravanti (5), and Blomquist (6) to require the presence of hyperplasia in the livers used for homogenate source. However, a requisite co-condition was a preexisting commitment to hyperplasia in the liver of the injected test animal. The same two requirements characterized the hepatic stimulatory substance found by LaBreque and Pesch (7) in regenerating adult and weanling rat liver cytosol. A heat-stable moiety of the cytosol was retained through several hundred thousand times purification $(1,8,9)$, and ultimately by the peptide product of the ALR gene (10).

Because ALR has no effect on either the resting intact liver, or on cultured hepatocytes (1), its identification through a succession of purification steps depended on two in vivo assays: an insensitive minimal hepatectomy model, in which ALR activity in an injected test substance was identified when it caused a greater than normal regeneration response to $30-40 \%$ hepatectomy in rats and dogs $(7,8,9)$, or, alternatively, with a canine Eck fistula model (11). With the Eck fistula assay, intraportally injected ALR produced a proliferative response beyond the low-grade hepatocyte hyperplasia produced with portacaval shunt in dogs. In addition, the ALR prevented the acute hepatocyte atrophy and dramatic organelle disruption that is produced by this operation $(8,9,11)$. The triad of mitotic response, organelle preservation, and prevention of atrophy in the Eck fistula model has successively defined a family of "hepatotrophic" agents beginning with insulin (12) and crude hepatic cytosol (11) that ultimately included recombinant ALR (10), three conventional growth factors (HGF, IGF, TGF $\alpha$ ) (1), two cell directed immunosuppressants (cyclosporine [13] and tacrolimus [14]), and the immunophilin $\mathrm{FKBP}_{12}$ (15). Why these diverse molecules all have essentially the same effects is an unresolved question.

Although the cDNA coding for the rat ALR has recently been isolated and a biologically active recombinant peptide produced (10), virtually nothing is known about the cellular physiology of ALR. One possible suggestion comes from the proven $50 \%$ homology that ALR protein shows to the product of the dual function nuclear Saccharomyces cerevisiae gene ERV-1, which is essential for oxidative phosphorylation, vegetative growth and life of the yeast (16). The availability of the mouse ALR gene should facilitate analysis of its role in liver and other organ physiology, as for example through construction of transgenic and knock-out animals. Knowledge of the human gene also should help delineate potential therapeutic implications.

We report here the complete (i.e., genomic) nucleotide sequence of the mouse ALR gene, as well as its mRNA transcript. We also report on ALR mRNA expression and its product presence in mouse tissues. In addition, we report the sequence of human ALR, and its comparison with the rat and mouse sequences. The human gene was found to map to human chromosome 16 . The mouse gene is located on a portion of chromosome 17, which has a documented homology to the human chromosome 16 , and where the $T / t$ region has also been mapped $(17,18)$. These findings imply a role for the highly conserved ALR gene, not only as a liver growth factor, but in a wide range of other biological processes as well.

\section{MATERIALS AND METHODS}

\section{Cloning of Mouse ALR Gene}

$\mathrm{A}(\mathrm{C} 57 \mathrm{Bl} / 6 \times \mathrm{CBA})_{\mathrm{Fl}}$ mouse genomic library in $\lambda$ FIX II vector (Stratagene, La Jolla, CA, U.S.A.) was screened with a rat ALR CDNA probe (i.e., 1.2-kb EcoRI fragment [10]). Phage plaques, plated at approximately 50,000 pfu/plate, were screened on duplicate filters by hybridization against a ${ }^{32} \mathrm{P}$-labeled rat ALR cDNA probe. Radioactive probes were generated by random priming using a Multiprime labeling kit (Amersham, Arlington Heights, IL, U.S.A.). Hybridization was performed according to Maniatis et al. (19). Positive plaques were purified by two additional rounds of filter hybridization. Restriction endonuclease digested DNA was fractionated by electrophoresis on agarose gels and transferred on Hybond $\mathrm{N}^{+}$membranes (Amersham). Blots were hybridized as in Giorda et al. (20).

\section{Mouse DNA Sequence Analysis}

DNA fragments were subcloned in pBlueScript II SK( $(-)$ plasmid (Stratagene). DyeDeoxi-sequencing reactions were performed using the PRISM DyeDeoxi Terminator Cycle Sequencing Kit (Applied Biosystems, Inc., Foster City, CA, U.S.A.) on a Perkin Elmer 9600 Thermal Cycler, and run on a Model 373A DNA sequencing system (Applied Biosystems). Sequence analysis was performed using the GCG programs (21) on a VAX 
computer at the Pittsburgh Supercomputing Center (Pittsburgh, PA, U.S.A.).

\section{Mouse cDNA Amplification and Sequencing}

Total cellular RNA was extracted from mouse testis and liver using RNA Stat-60 (TEL TEST "B", Inc., Friendswood, TX, U.S.A.) following the manufacturer's instructions. First strand cDNA was synthesized using oligo-dT primer and a Gen-Amp RNA PCR Kit (Perkin Elmer, Chicago, IL, U.S.A.). Polymerase chain reaction (PCR) amplification was performed using the same kit on a Perkin Elmer 9600 Thermal Cycler. Five-prime and 3' RACE were performed using 5' and 3' RACE systems (Gibco BRL, Bethesda, MD, U.S.A.) following manufacturer's instructions, with the following modifications. First strand CDNA was generated in the 5' RACE reaction using oligo-dT as primer. PCR products were cloned in pBlueScript II SK(-) and sequenced as described above.

\section{Northern Blot Analysis in the Mouse}

A mouse Multiple Tissue Northern blot filter (Clontech Laboratories, Palo Alto, CA, U.S.A.) was hybridized with ${ }^{32} \mathrm{P}$-labeled cDNA probes following manufacturer's protocols, washed at high stringency, and exposed to Kodak XAR films.

\section{Chromosomal Mapping of Mouse ALR}

Chromosomal location of the gene was determined by hybridization of the radiolabeled mouse ALR probe to HindIII digested genomic DNA from a [C57BL $\times M$. spretus] $\times M$. spretus backcross panel of 96 animals (Jackson Lab Backcross DNA Panel Map Service, Bar Harbor, ME, U.S.A.). Linkage analysis was performed by the Jackson Lab Backcross DNA Panel Map Service.

\section{Western Blot Analysis of Mouse ALR Protein}

Crude protein extracts from rat liver and mouse testis, liver, and spleen were prepared as described previously (22). Soluble and insoluble proteins were separated by centrifugation at $15,000 \times g$ for $30 \mathrm{~min}$ at $4^{\circ} \mathrm{C}$. Protein concentration was determined by Bradford assay. One hundred milligrams of each sample were loaded on a $12 \%$ SDS-PAGE gel, and transferred on PVDF membranes (Immobilon-P, Millipore, Marlborough, MA, U.S.A.) according to Sambrook et al. (23). Transfer efficiency was determined by staining with Ponceau S. The filters were blocked for $1 \mathrm{hr}$ in Transfer-Tris Buffer Solution (TTBS) at room temperature, incubated with anti-ALR antiserum $(20 \mu \mathrm{g} / \mathrm{ml}$ in TTBS $)$ for $1 \mathrm{hr}$, washed four times for $5 \mathrm{~min}$ in TTBS, incubated with peroxidase enzyme (PE)-linked donkey anti-rabbit Ig (1: 3,000 in TTBS) (Amersham) for $30 \mathrm{~min}$, washed again in TTBS, and developed with $\mathrm{DAB} / \mathrm{NiCl}_{2} / \mathrm{H}_{2} \mathrm{O}_{2}$ in $0.1 \mathrm{M}$ Tris- $\mathrm{Cl}$ ( $\mathrm{pH}$ 7.5). Incubation with second antibody only was performed as a negative control. Anti-ALR N-terminal antiserum was prepared by Dr. A. DeLeo of the Pittsburgh Cancer Institute by immunizing a rabbit with repeated injections of the synthetic peptide MRTQQKRDIKFRED (Fig. 2).

\section{Cloning of Human ALR cDNA}

Twenty million plaques from a human hepatoma cell line HepG2 cDNA library (Clontech Laboratories) were screened with the rat ALR cDNA as a probe as described for mouse ALR gene cloning. Positive plaques were purified and their DNAs subcloned in pBlueScript II SK (-) (Stratagene), analyzed by restriction enzyme mapping, and sequenced as described above.

\section{Northern Blot Analysis in Humans}

A human Multiple Tissue Northern blot filter (Clontech Laboratories) was hybridized as described above.

\section{Chromosomal Mapping of Human ALR}

A primer pair that would specifically amplify a 230-bp fragment of the human ALR cDNA was used to amplify genomic DNA from the NIMGS Human/Rodent somatic cell hybrid mapping panel \#2 (Coriell Cell Repositories, Camden, NJ, U.S.A.). Primer sequences were: Primer A: 5'ACC CAG ACA CCC GCA CC-3'; Primer B: 5'GCT GTG ATG CAC TTT AAT GAG-3'. Amplification conditions were: $2 \mathrm{mM} \mathrm{Mg}^{++}$in PCR buffer I (Perkin Elmer), $0.2 \mathrm{mM}$ dNTPs, $0.2 \mu \mathrm{M}$ primers. Reactions were performed in $25 \mu \mathrm{l}$, for $30 \mathrm{cycles}$ of $20 \mathrm{sec}$ at $94^{\circ} \mathrm{C}, 30 \mathrm{sec}$ at $58^{\circ} \mathrm{C}, 30 \mathrm{sec}$ at $72^{\circ} \mathrm{C}$, on a Perkin Elmer 9600 Thermal Cycler. 


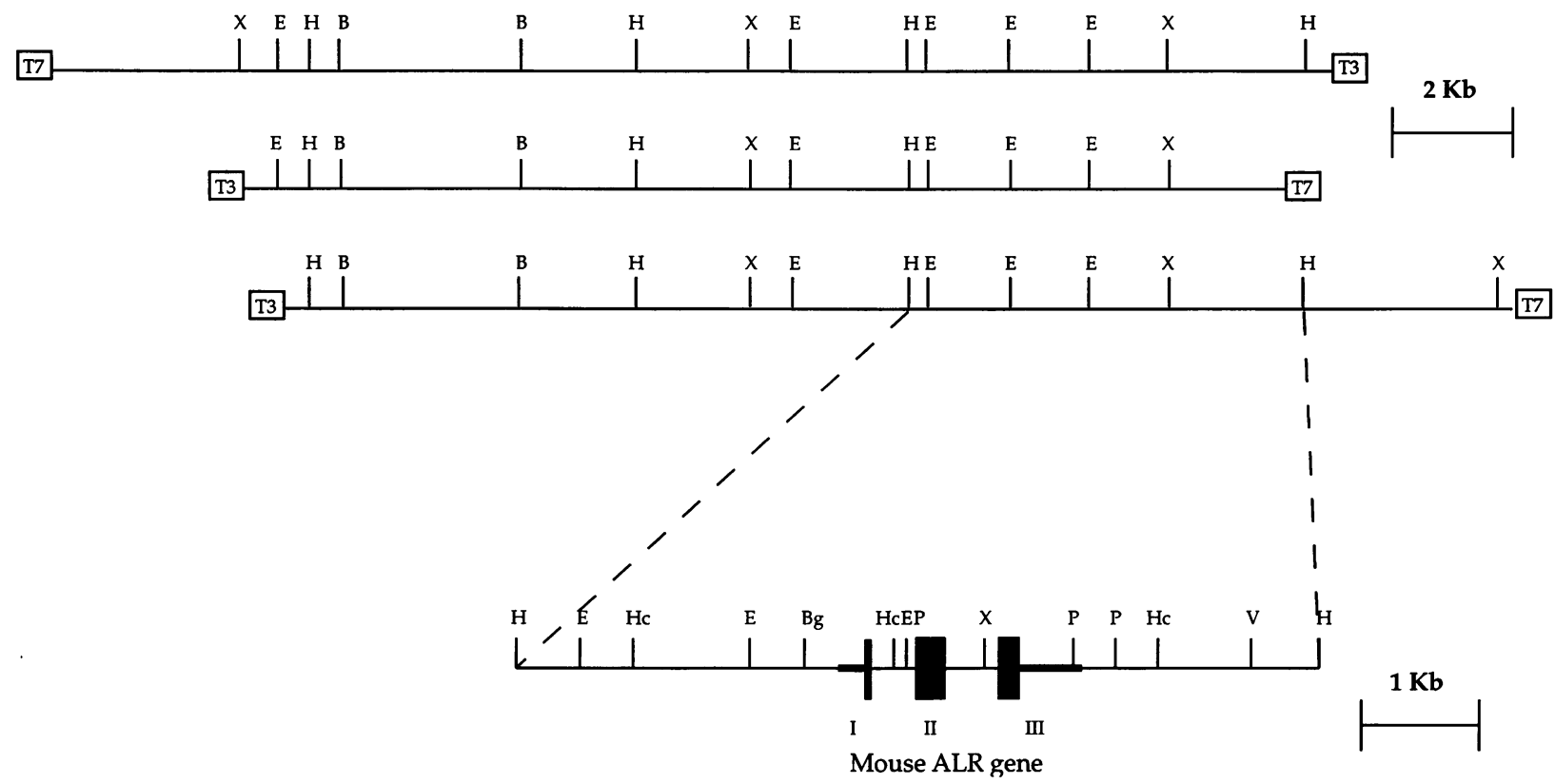

FIG. 1. Genomic organization of mouse ALR

A partial restriction map of the three aligned genomic ALR clones is shown. The HindIII fragment containing the ALR gene is shown in further detail. Restriction sites are: X, XbaI; E, EcoRI; H, HindIII; B, BamHI; P, PstI; Hc, HincII; Bg, BglII; V, EcoRV. The protein coding portions of the gene are indicated as black boxes. The 5' and 3' nontranslated portions of the transcript are shown as thick lines.

\section{RESULTS}

\section{Genomic Organization of Mouse ALR}

Screening of a $(\mathrm{C} 57 \mathrm{Bl} / 6 \times \mathrm{CBA})_{\mathrm{F} 1}$ (mouse) genomic library with a rat cDNA probe yielded three positive clones ranging in size from 17 to $22 \mathrm{~kb}$. The aligned inserts cover a region of about $25 \mathrm{~kb}$ (Fig. 1). By Southern blot hybridization with the same rat ALR cDNA probe, it was demonstrated that the entire ALR gene is contained in a $6.7-\mathrm{kb}$ HindIII fragment, which was sequenced in its entirety (Fig. 2).

The exon/intron organization was elucidated initially by comparing the mouse genomic sequence of the HindIII fragment to the previously published rat cDNA sequence, and then aligned with the mouse cDNA sequence, subsequently obtained from $5^{\prime}$ and $3^{\prime}$ RACE analysis. The protein coding portion of the gene, previously defined by Hagiya et al. (10), was found to be coded by three exons: the first exon contains both the $5^{\prime}$ untranslated sequence, and 18 bases following the ATG translation initiation codon. The second exon is of 198 bases, and the third exon contains the remaining portion of the protein coding sequence, followed by the entire $3^{\prime}$ untranslated sequence. These three exons are separated by introns of 400 and 480 bp respectively (Figs. 1 and 2). A 500-bp G/C-rich region is located almost immediately upstream of the translation start codon. The closest sequence resembling a

\section{FIG. 2. Genomic sequence of the mouse ALR gene}

The portion between bases 1,351 and 5,000 of the HindIII fragment is shown. A Bl repeat-like sequence is at bases 1-160 shaded in grey. Putative TATA boxes are underlined. The start of the rat cDNA is indicated by a dot. The start of the mouse cDNA is indicated by a black triangle. Potential ATG sites are emphasized by a black background. The putative protein coding regions are translated in single letter amino acid code (letters in bold). The polyadenylation signal sequence is boxed. The end of the mouse transcript is indicated by a black triangle. Conventional primers (grey arrows) used for mouse cDNA amplification are indicated with capital letters Al (bases 1-17; sense), B1, and B2 (bases 257-239 and 683-666, respectively; both antisense). The entire HindIII sequence has been deposited on GenBank (Accession number: U40496). 
1351

GTTCTCAGTGGGGT AGCACATGCCTTTATTCCCAGCACTTGGGTGGCAGAGGCAGGTAGATTTCTGAGATCAAGGCCAGCC TGGTCTACAAAGAGAGTCCAGGACAGTCAAGACTGGTTACACTAAGAAACCCTGTCTTGATTTGCAC ACACACACACACACACACACACACAAATCCACTGTCCCAAATTGCCCAACATCTCTGTTTTTAGGGA AAAAAATGCCAGTTGGTACCATGGTCATAAGTAGGCCTTGCCGGACTCTGGATTCTGCTCTATCTCC TCTACCTTCCCTAGACCCTGCCCCCAGCGTCACTGGTGTTTCTGTAAAATGTCAAGCATTTGAGCTG ATTTCTTATCCCAAAGGCCTGTGCCTAAGGGTCTCCTCACCCTCTGCACAGCTTTCATCAGTTTTGT AAATATGATTCTGGTGTACTGGGTTTTCCGCATTTGCATCACTGGTTAAAGGCTAAAGAGGGAGGCT TGTTCCTTGTTCTGCTCAGTGGAGGAAACAGCACCTGGAGAGAGCAGCCACTCTACAAATATTTGCA GAAAGAGTAAATGAACACATGGAGGCAGCGGAAGGAATTCAACATCATCTTCACTCAGCTACATATA GAGTTCAAAGTTAGTCCAAGTTACATGAGACCCTGTCTCCAAAAGAAAAAAAAAAGGAAGAAATTCC TGAGGCTGCTATTAAAATAGGCATAATAATCTTTGATCCAAGCTAGAGGATGAGGACACGCCCTCTC TCAAGGTTGCGCAGTGGGGGACAGTTACTAGGGCACAGGTAGCCTCTGGCAATAAGTACTGTCTGGT CTCTGTGAGGCCACGCCCCGTCGCGCGTTGCTAAGGCACCGAGAAGCGTGTAGGTGGGCACGTCGTA AGGCAGGAAGACCCCAAATTTTGCCGCCCTTAGGTGTCTGAGCCAAGCGATGGGGGTGATGCGAGAA GCATCTCGGAACTCATCTCAGTCCAAGATCTCAGCACAGCCGCAGCGCGCCTTCCACGACACACTTC ACATCTGCCGGAGGAAGCCTGAACCCCACCATGGACCACGCACGCCGGGCTCGCGGGGGGTCACCAG GCGCAAGAGTACCCGCAGAGCCGGAGCCGCAGCCGCCAGTGCCGCCTCGGCCTGGGCCTCCGGGGTC GTCTCCTCCCGCCCCGAGGTCTCCTACGACTCCGCGAACCGGGCCGCCGACTCGGnCCCCAGTCCCG CCCCGCCGCCAGGCCCCGCCTCCTGCCCCGGnCTGAGCCCAGCCCCGGGCTGCGGTTTCCGCTCCTn CCACCGCACGCCGGCGGTGGCGTGCACGCTCCTCCGAACTGTCGCCGGCACGCCCTCTTGGCCCCGC A1 TGCCCGCACGCCAGCAAGATGGCGGCGCCCAGCGAGCCGGCGGGCTTCCCTCGCGGCAGTCGCTTCT CCTTCCTGCCGGGCGGCGCGCGCTCCGAGATGACCGACGACCTGGTGACTGACGCGCGGGGCCGCGG CCCAAGGCATAGAGACGACACCACCCCTTCCGCGGCCCCGGCGCCGCAAGGTTTGGAGCACGGGAAG CGACCGTGCCGGGCCTGCGTGGACTTCAAGTCGTGGATGCGGACCCAGCAGAAGGTGCAGATGCCCC B1 $M$ R T \& $Q \mathbf{K}$

GCTTGGCCGCCCGGTCCCGGTCCCCCACACACCTGGTTTCCAGGTTCCCGTTCGGCTACCGCACGGC CCAAGCCCTCAGGGTTGGCTATGCCTAAACATGTTTACCCCTCACCCACACACACCGTTAGGCTCAA ACTTTTGCGCTTTCAGCCACTGGCTAGTCAGATCGAGTTAGTAGGCTGTCAACTGAAAAAGGGAGCG TTCTTGTTTCCTTTATTATTATTATTATGATTATTATTATTATTATTATTATTATTAGTGATAATGG GAATTCGAACCGAAGGCCTCTGGCGTACAACGCAGGCAGTCTGCCACTGGGTTGGAATTTTATGGGC TTTCTGGTTGGGTAAGAGTGAATTGACCTTTAGTCGGCCTACTTCTGCAGCGGGACATCAAGTTTAG

$\begin{array}{lllllllll}R & \mathbf{D} & \boldsymbol{I} & \boldsymbol{K} & \boldsymbol{F} & \mathbf{R}\end{array}$ GGAGGACTGTCCGCAGGATCGGGAAGAATTGGGTCGCCACACCTGGGCTTTCCTCCATACGCTGGCC $\begin{array}{llllllllllllllllllllll}\mathbf{E} & \mathbf{D} & \mathbf{C} & \mathbf{P} & \boldsymbol{Q} & \mathbf{D} & \mathbf{R} & \mathbf{E} & \mathbf{E} & \mathbf{L} & \mathbf{G} & \mathbf{R} & \mathbf{H} & \mathbf{T} & \mathbf{W} & \mathbf{A} & \mathbf{F} & \mathbf{L} & \mathbf{H} & \mathbf{T} & \mathbf{L} & \mathbf{A}\end{array}$ GCCTATTACCCGGACAGGCCCACGCCAGAACAACAACAGGATATGGCCCAGTTCATACATATATTTT $\begin{array}{llllllllllllllllllllll}\mathbf{A} & \mathbf{Y} & \mathbf{Y} & \mathbf{P} & \mathbf{D} & \mathbf{R} & \mathbf{P} & \mathbf{T} & \mathbf{P} & \mathbf{E} & \mathbf{Q} & \mathbf{Q} & \mathbf{Q} & \mathbf{D} & \mathbf{M} & \mathbf{A} & \mathbf{Q} & \mathbf{F} & \mathbf{I} & \mathbf{H} & \mathbf{I} & \mathbf{F}\end{array}$ CCAAGTTTTACCCCTGCGAGGAATGTGCGGAAGACATAAGGAAGAGGTAAGTTGTGGGTATACCTCT $\begin{array}{llllllllllllllll}\mathbf{S} & \mathbf{K} & \mathbf{F} & \mathbf{Y} & \mathbf{P} & \mathbf{C} & \mathbf{E} & \mathbf{E} & \mathbf{C} & \mathbf{A} & \mathbf{E} & \mathbf{D} & \mathbf{I} & \mathbf{R} & \mathbf{K} & \mathbf{R}\end{array}$

GTCAGTAAGGTCCCTAGAGCTGGCCTAGCTGATGCCGGGCGGTGGTGGCGCACGCACCCAGCACCTG GGAAGCAGTGGCAGGTGGTTTTCTGAGTTCCGAGGGCAGCCTGGTCTTCAAAGTGAGTTCCAGGACA GCAGGGCTACACAGAGAACCCCCTGTCTCGAGAAACCAAAAAAAAAAAAAAAACTGGACTGAAGTAA ACATCCCTCACTCTCAGTGGCCTAACCTAGGTTGCACTTGTTATTTGAAGCAGTTAGGAGTGAGGGG AGCTCTAGCTGCATTGTGCCCCTGGTGCTGAGAAACAGTCCTTTGTGCCAGGCCCTACCTAGAAACT CTAGATTCAGGCCCCCCCACCCCCTACAGGAAGATAGGTCATAGCAGTGCCCCAACTCCCGTCTCCA GACACTGGCAGAGACAGAGGGGGCGGGATCTCCTTACATCCTTCTGTGCTTCTCTCAAAGGATAGGC I $\mathbf{G}$ AGGAACCAGCCAGACACAAGCACTCGAGTATCCTTCAGCCAGTGGCTGTGCCGCCTGCACAATGAGG $\begin{array}{llllllllllllllllllllll}R & \mathbf{N} & \boldsymbol{Q} & \mathbf{P} & \mathbf{D} & \mathbf{T} & \mathbf{S} & \mathbf{T} & \boldsymbol{R} & \mathbf{V} & \mathbf{S} & \mathbf{F} & \mathbf{S} & \boldsymbol{Q} & \mathbf{W} & \boldsymbol{L} & \mathbf{C} & \boldsymbol{R} & \boldsymbol{L} & \mathbf{H} & \mathbf{N} & \mathbf{E}\end{array}$ TGAATCGGAAGCTGGGCAAGCCTGATTTTGACTGCTCGAGAGTAGATGAGCGTTGGCGTGACGGATG

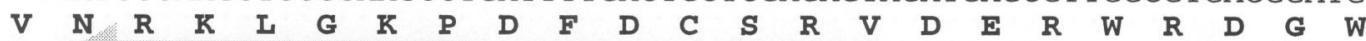

B2 GAAGGACGGCTCCTGTGACTAGAAGATTACCAGCAGTTCGGGAGGGGGATCTAGGCTGGTTCTATGG K D G S C D *

GCAACAGCCTGATTGACGATTAAAGTGCATCTGAGCCAACACTTGTTTCTGTAGTTGGGGAAGAAAT CCCTAGAACACTGCCTCTGGGAACCCTGCCACAGGATTAAGAAATGGAGGTGCCTACTACAGACACn TGGGTGCCCTCCTCAGCGAAAGCCCCATGAGACTGAAGGTCAGAGGCAGGAGTGGTCCAGGTGCCTC GTACCCTTCATTTGGGACATAGTTGCCCCAAAGGAAGATGGACTAGATGATTGCCTTACTACTACAC AGCCCTGGCTGCCTACAAGAACTGTGATCCTGGCAACTCTnCCATTTCAGAATTGAAGCAGGGTTCC CCAGCTCCAGCAGGGTTTTTCTCTTAGGGTGTACCAACCATGGTGCCCACTGTCAGCCTGGCACATG GTCTTCTGCAGCTATGACAAGCATGTCAGCAGAGGGTCCTGGGAACAGCTTCCTTAGCGTTTGAGGC CAAAATAAACTGAAGTGACTTAGGGGCTGGTTTCTGTGTGCTTTGTGTTTGGGCTTACAGTGGTGGA CTGGCTCGGTTTGGCCTGTCTGTGATTAGTTGGGCCCTCTTGTCTGGAGTTGGCAGAAGTCACCATG GTGCAGCTGAGACATTTCAGGGAGTGTCAGAATTTCCAGGGTGATGAAGAAGCTGAAGAAGACTAGG GGTCTGTGGGGGTACAAATGGGGAGTGGTCAGAGTGTCGCCAGCTGGGATTGTGTCTCGGTGAAGTT GAGAACTGCTGAGTATAA 
MALR

rALR

hALR

MALR

rALR

hALR

MALR

rALR

hALR

MALR

rALR

hALR

mALR

rALR

hALR

MALR

rALR

hALR

MALR

YALR

hALR

MALR

YALR

hALR

MALR

rALR

hALR

MALR

rALR

hALR

MALR

rALR

hALR

MALR

rALR

hALR

MALR

rALR

hALR

MALR

rALR

hALR

MALR

rALR

hALR

MALR

rALR

hALR

MALR

IALR

hALR

MALR

rALR

hALR

MALR

IALR 1049

hALR

rALR

hALR

MALR

rALR

461

516

515

521

576

575

581

641

696

693

$1 \ldots \ldots \ldots \ldots \ldots \ldots$ C GCACGCCGGCGGTGGCGTGCACGCTCCTCCGAACTGTCGCCGGCACGCCCTCTTGGCCC 1 C GCGCGCTGECGGTGGCATGCGCGCT GCTCT G T C G TCTCCTGCACGCCCTCTTGGCC

$1 \ldots$

$61 \mathrm{CGCTGCCCGCACGCCAGCAAGatggcggcgcccagcgagccggcgggct} t c c c t c 9 c g g c$

61 C G T TCTCG TACGCCA GCAAT A T G GCD

A A C C TCTTC - - T TCCTGCCGGGGGGCGCGCGCTCCGAGATGATGGACGACCTGGCGACC

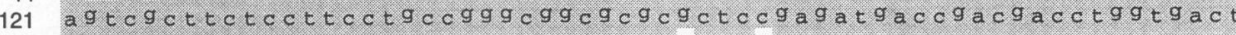

121 A GTCGCT TCTCCT TCCTGCCGGGCGGCGCGCACTCGGAGATGACCGACGACCTGGTGACT

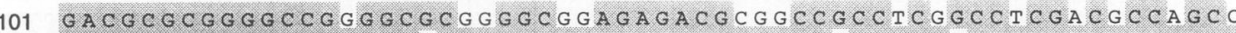

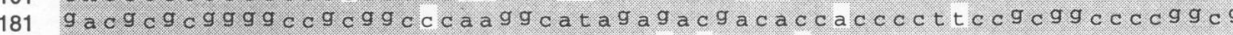

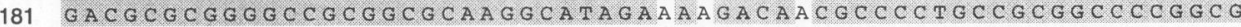

CAGGCGCCGAC C T C C GA T T C T C C T T T G C C G G G A C C C T C C C G A G G C G GC C T T C C C

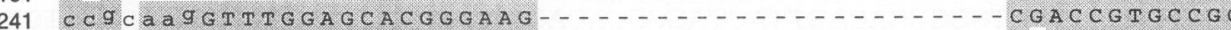

241 C CGAAAGGTTTGGAGCACG G GAAG $\ldots \ldots \ldots$

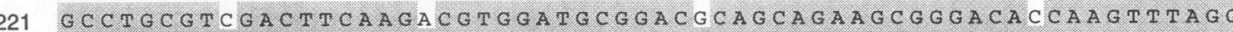

277 GCCTGCGTGGACTTCAACTCGTGGATGCGGACCCAGCAGAAGCGGGACATCAAGTTIAG

276 GCCTGCG TGGACTTCAAGTCGTGGATGCGGACCCAGCAGAAGCGGGACATCAAGTTTAG

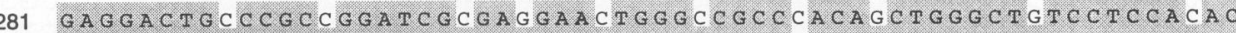

337 G A G G ACTGTCC GCA G A T C G G A A G A T T G G T C G C C - A C A C C T G G G C T T TC C T C A T A

336 GAGGACTGTCCACA G A TCGGGAAGAATTGGGTCGCA-ACACCTGGGCTTTCCTTCATAC

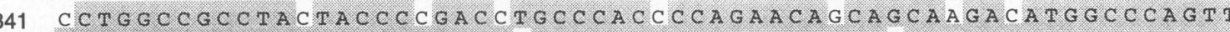
396 GCTGGCCGCCTATTACCCGGACAGGCCCACGCCAGAACA ACA ACAGGATATGGCCCAGT

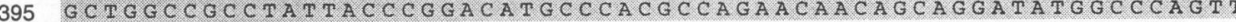

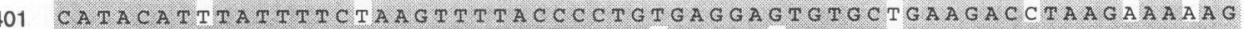
456 C A R A ATATATTTTCCAAGTTTTACCCCTGCGAGGAATGTGCGGAAGACATAAGGAAGA 455 CATACATATATTTTCCAAGTTTTACCCCTGTGAGGAGTGTGCAGAAGACATAAGGAAGAC

636

701

755

815

810

T T G T G CA G A A C C A C CA A A A C C G CACCCGGGCATGCTTCACACAGTGGCTGTGCCA

G ATAGGCAGGAACCAGCCAGACACAAGCACTCGAGTATCCTTCACCCAGTGGCTGTGCCC

GA $T A G A C A G G A G C C A G C C A G A C A C A A G C A C T C G A G T G T C C T T C A G C C A G T G G C T G T G C C G$

C CTGCACAATGAAGTGAACCGCAAGCTGGGCAACCCTGACTTCGACTGCTCAAAAGTGGA C C T G CACAATGA G G T A A TCGGAAGCTGGGCA A G C T G T T T T GACTGCTCGAGAGTAGA C C T T CACAATGAAGTGAACCGGAA GCTGGGCAAGCCTGATTTTGACTGCTCAAGAGTTGA

$T G A G C G C T G G C G C G A C G G C T G G A A G G A T G G C T C C T G T G A C T A G A G G G T G G T C A G C C A G A G$ TGA GCGTTGGCGTGACGGATGGAAGGACGGCTCCTGTGACTAGAAGATTACCAGCAGTTC T A A C G A T GC T T ACGGCTGGAAGGACGGCTCCTGTGACTA-AGGATTACCA-CAGACC

CTCATGGGACA G TAGCCAGGCATGGTTGCATAGGGGCAGGGCACTCATTAAAGTGCATC G GGA GGGGGATCTAGGCTGGTTCTATGGGCAACAGCCTGATTGAC-GATTAAAGTGCATC GTGCAGGGCAACGC CGG-G-TTCTATGGGCAACAGCCTGACTGAC-GATTAAAGTGCATC

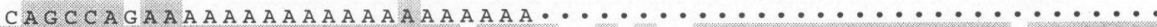
TGAGCCAACACTTGTTTCTGTAGTTGGGGAAGAAATCCCTAGAACACTGCCTCTGGGAAC TGA GCCAAA GCTTGITTCTGTGGTGGGGGTGGGATCCCCTAGAACACTGCCTATGGGAAC

$\ldots \ldots \ldots \ldots \ldots \ldots \ldots \ldots \ldots$ C CTACCCACAGACTCAGAAACGGAGGTGCCCACTATAGACAGTTGGGTGGCTTCCTCAGG

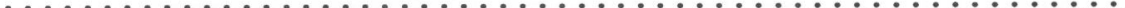
875 G- - A A A GCCCCATGAGACTGAAG GTCAGAGGCA G GAGTGG TCCAGGTGCCTCG TACCC 870 TCTTAAAGCCCCATGGGACTGAAGATGAGAGGCAGGAGTGGTCCAGGGCACCCCATACCC

$\ldots \ldots \ldots \ldots \ldots \ldots \ldots \ldots \ldots \ldots \ldots$ $931 \ldots$ 930 СТTATGATAC С С T TATACATTTGGGACATAGTTGCCTCAAAGGAAGGTGGGCTAGAC CA $\ldots \ldots \ldots \ldots \ldots \ldots \ldots \ldots \ldots \ldots \ldots \ldots \ldots \ldots \ldots \ldots \ldots \ldots \ldots \ldots \ldots \ldots \ldots \ldots \ldots \ldots \ldots \ldots \ldots$ 975 T TGC CTTACTACTACACAGCCCTGGCTCCCTACAAGAACTGTGATCCTGGCAACTCT RCC 990 IT IC CTTC C TACTACATATCCCCA GCTGCCTACA-GAACTGTGACCCAGGCA ACTCTGCC

$\ldots \ldots \ldots \ldots \ldots \ldots \ldots \ldots \ldots \ldots \ldots \ldots \ldots \ldots \ldots \ldots \ldots \ldots \ldots \ldots \ldots \ldots \ldots \ldots \ldots \ldots \ldots \ldots \ldots$ 035 A TTTCA A A TTGAAGCA G G T TCCCCAGCTCCAGCAGGGTTTTTCTCTTAGGGTGTACCA 49 A TTTCAGAATTGAAGCAGGGTTCC--AGCTCTAGTTGGGTTTTTCTCTTAGGGTAAACCA

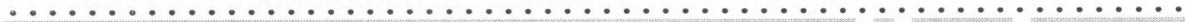
1095 A C CATGGTGCCCACTGTCAGCCTGGCACATGGTCTTCTGCA GCTATGACAAGCATGTCAG 1107 ACCATGGTGCCCACTGTCAGCCTGGCACATGGTCTTCTGCAGCCAGGACAAACATGTCAC

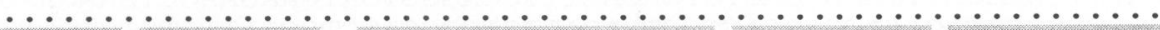
1155 CAGAGGGTCCTGGGAACAGCTTCCTTAGCGTTTGAGGCCAAAATAAACTGAAGTGACTT 1167 CAGAGGATCCTGGGAAGGGCTTCCTTAGCGTTTGAGACCAAAATAAAATGAAGTGACTT
43

120

120

100 180

180

160 240

240

220 276

275

280

336

335

340

395

394

400

455

454

460

515

514

520

575

574

580

635

634

640

695

692

700

754

728

814

809

874

869

930

929

974

989

1034 1048 


\begin{abstract}
hALR
MALR

YALR

hALR

MALR

YALR

hALR

MALR

YALR

hALR

MALR

rALR

hALR

MALR

rALR

B

FIG. 3. Comparison of human, mouse, and rat human ALR

(a) Alignment of human, mouse and rat cDNA sequences. Spaces introduced to maximize the identity are indicated by dashes. The portion of sequence absent in the mouse cDNA is shown in lower case letters. Dots indicate absence of the base. (b) Alignment of putative ALR proteins translated from cDNA. To maximize the amino acid alignment the " $\mathrm{C}$ " at position 318 of the human sequence was omitted before translation and may be a sequencing error. The ATG used as translation start codon in the rat sequence (10) is also used as translation start for the mouse and human sequences.
\end{abstract}

TATA box motif is located over $550 \mathrm{bp}$ upstream of the first base of the rat cDNA. Regions containing sequences very similar to Alu-like Bl repeats are present both upstream and downstream from the ALR gene (Fig. 2) (24).

\section{Expression of Mouse ALR}

The structure of the mouse ALR transcripts was analyzed by $5^{\prime}$ and 3' RACE. As expected, rat and mouse cDNAs were found to be highly homologous in both nucleotide sequence ( $90 \%$ identity) and putative translation product ( $96 \%$ identity). The human cDNA was also found to be very similar to the rodent cDNAs, particularly at the protein level (Fig. 3). Polyadenylation of human and mouse mRNAs appears to occur at the same position as that seen for the rat mRNA. The $5^{\prime}$ RACE amplification generated a fragment starting at position 49 of the rat cDNA. Remarkably, a 166-bp portion of mouse cDNA sequence, corresponding to position $82-248$ of the rat cDNA, was found consistently to be missing both in RACE protocols and in amplifications performed with conventional primers (Fig. 2). The exon/ intron boundaries of this mouse intron, unlike the human and rat sequences, were found not to contain canonical AG_GT motifs.

By Northern blot hybridization of the ALR
cDNA as probe to a panel of poly- $\mathrm{A}^{+}$RNAs from mouse tissues, a 1.3- to $1.4-\mathrm{kb}$ band was found in mRNA from all mouse tissues examined, but liver, and particularly testis, yielded the strongest signal (Fig. 4). By sequencing, ALR transcripts from both liver and testis have the same structure. The ALR transcript in the liver was found not to show a demonstrable change in quantity during the tissue regeneration that follows partial hepatectomy (data not shown).

From this analysis of the gene sequence and transcript size, it was not possible to determine the ALR protein's size and structure. Therefore, we attempted to determine directly the precise size and structure of the ALR protein by Western blot analysis with a rabbit antiserum against a putative $\mathrm{N}$-terminal peptide of the rat protein. We found this serum to recognize a $23-\mathrm{kD}$ band in all tissue examined (Fig. 5). The amount of 23-kD protein was found to be highest in testes, which also yielded a band of higher molecular weight of approximately $38-40 \mathrm{kD}$. We found this $23-\mathrm{kD}$ band to be present in both the soluble and insoluble fractions of the testes lysate. Rat liver was also found to contain a band of $23 \mathrm{kD}$, although the majority of ALR seen on the gels was present in a band of approximately $28 \mathrm{kD}$. Larger positive bands were found to be present in lysates of both testes and liver, and this may 

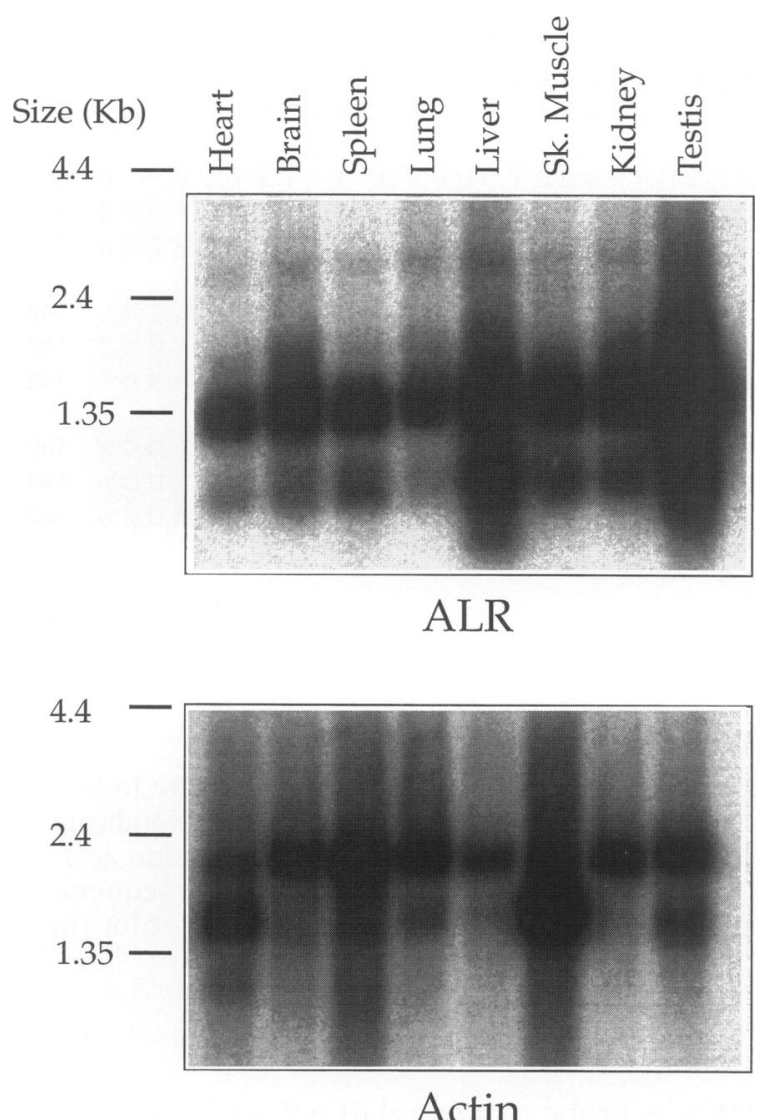

FIG. 4. Expression of ALR in mouse tissues

The same filter was first hybridized to ALR cDNA (upper panel), then stripped and hybridized to mouse actin (lower panel). The size of RNA molecular weight markers are indicated. A Phosphorimager SI (Molecular Dynamics, Sunnyvale, CA, U.S.A.) was used to directly read these filters. indicate association of a fraction of the ALR protein with other cellular proteins.

\section{Mapping of Mouse and Human ALR}

By Southern blot analysis, we found that mouse ALR is present as a single gene copy (data not shown). The chromosomal location of the ALR gene was determined by hybridization to a [C57BL $\times M$. spretus] $\times M$. spretus backcross panel, and found to be located on mouse chromosome 17, in close linkage with the marker D17Mit55. We also determined the chromosomal assignment of ALR human homolog by utilizing a panel of monochromosomal hybrid cell lines. Primers for a portion of the 3 ' -untranslated sequence of human ALR amplified a band of the expected size only from the sample containing chromosome 16 (data not shown). The mouse ALR gene is thus located in a portion of chromosome 17 with homology to human chromosome 16 (Fig. 6). These results are in agreement with the current information on mouse chromosome 17 (25), and place the ALR gene in a clearly defined position of the mouse map.

\section{DISCUSSION}

All the presented results certainly shed some new light on our understanding of the ALR gene structure and expression. However, numerous specific questions about ALR remain unan-
FIG. 5. Western blot analysis of ALR protein in mouse tissues

Tissue lysate (L), cleared supernatant (S), and pellet of insoluble material (P) were analyzed from testis, liver, and spleen. Rat liver lysate was added as a control. The size of protein molecular weight markers are indicated. (left) Incubation with anti-ALR antiserum. (right) Incubation with second antibody alone (only a representative subset of the samples was incubated with second antibody alone).

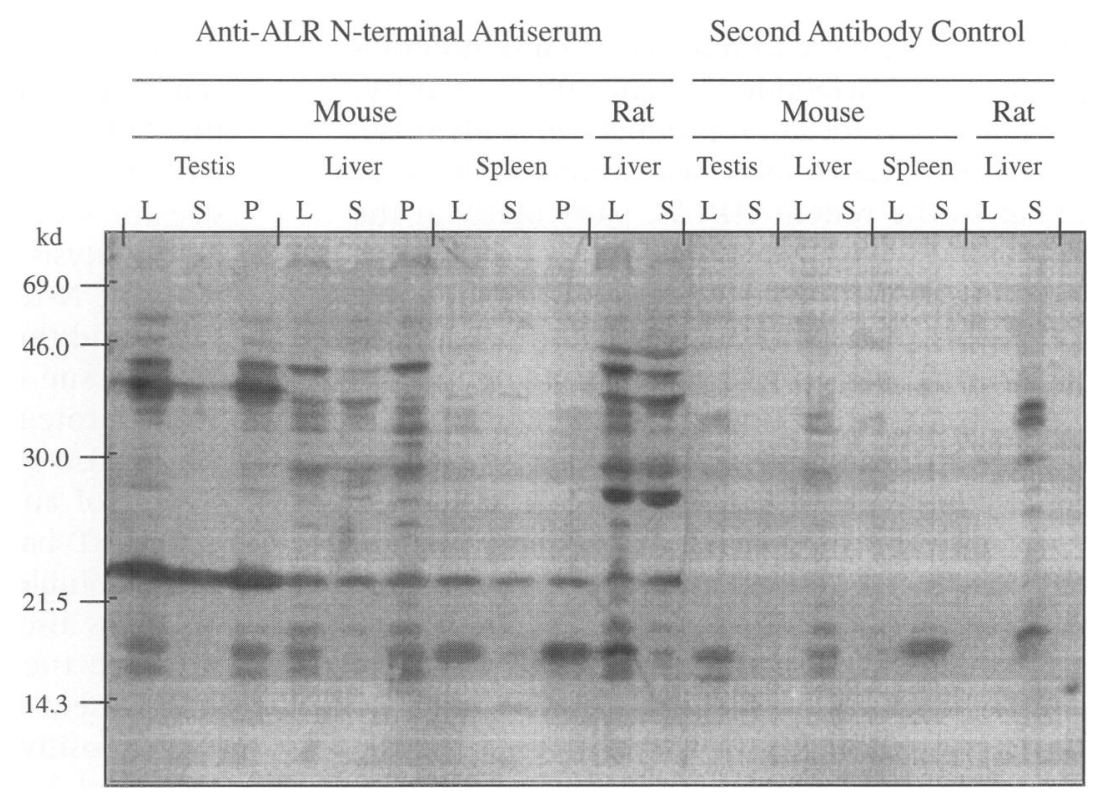




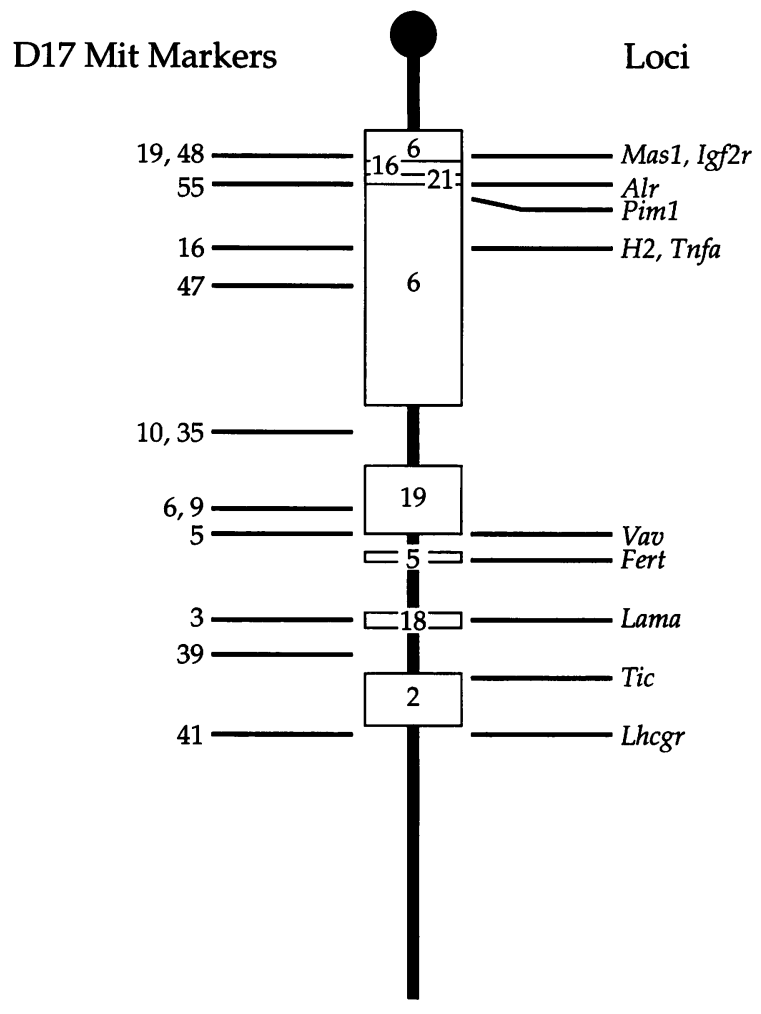

FIG. 6. Chromosomal location of mouse ALR

The location of known marker genes is indicated on the right. The location of random polymorphic markers is shown on the left. The location and approximate size of portions of the chromosome with linkage conservation between the mouse and human genomes are shown and numbered with the number of the corresponding human chromosome. Adapted from Ref. 25.

swered, including the size and amino acid sequence of the peptide and its physiologic function.

Rat ALR cDNA was originally described as having only one ATG translation start codon in the correct reading frame (10). Later, a correction in the noncoding cDNA sequence generated a longer open reading frame containing two additional ATG codons upstream from the original ATG (26). The same three in-frame ATG codons are also present in the human ALR cDNA, while in the mouse the observed 166-bp deletion removes both additional ATGs. However, while the absence of this segment of the mouse gene was observed consistently, we cannot exclude that this absence could be an artifact of the amplification procedure that is caused by the somewhat peculiar sequence of the region, particularly in view of its unusual exon/intron junction.
If we ignore the deletion, the genomic ALR sequence reveals an even longer open reading frame, containing two additional ATG codons. Given the size of the mouse ALR transcript, 1.3$1.4 \mathrm{~kb}$, only $100-200$ bases of upstream sequence can be transcribed in addition to the known sequence. If the TATA box of the ALR promoter is located in the position indicated in Fig. 2, one or more additional introns must be postulated to account for the size of the mature transcript. In addition, the faint $2.7-$ to $2.8-\mathrm{kb}$ band visible in the Northern blot in Fig. 4 could indicate the size of the primary ALR transcript, placing the transcription initiation site in close proximity to the putative TATA box. Unfortunately we have not yet been able to obtain a longer 5' RACE product, most likely because of the presence of the G/C rich content of this region.

The size of the ALR protein determined by Western blot argues for a translation product larger than the one previously postulated (10). Two major 45- and 28-kD bands were visible in rat liver. The latter band is not present in the mouse, and its size is close to that previously described for the protein in nondenaturing conditions. Based on the revised cDNA sequence (26), we would expect to deal with a protein of $22.8 \mathrm{kD}$. SDS-PAGE under the reducing condition showed ALR proteins with 23 and $14 \mathrm{kD}$, which suggest the possibility that the rat ALR is physiologically a homodimer. Thus, the ALR gene may have multiple translational initiation sites, or alternatively, ALR protein may be processed by a post-transcriptional mechanism.

As in the rat, mouse ALR is expressed in all tissues analyzed, with testis having the highest levels of both RNA and protein. Regenerating liver does not appear to express markedly higher levels of ALR mRNA, as assessed by the conventional Northern blot analysis shown here, although a more quantitative Northern blotting showed that the mRNA level increased at least two times at $12 \mathrm{hr}$ after partial hepatectomy, and kept the same level to $24 \mathrm{hr}$ (Dr. Okita, Yamaguchi University, Japan, personal communication). The source of the ALR protein and its dramatically increased plasma levels during liver regeneration (1) remains, then, to be fully elucidated.

Although the discovery of ALR stemmed from investigations of liver regeneration, preoccupation with this narrow physiologic context is no longer justified. The sequence of ALR is remarkably conserved between the mouse and rat to human, particularly at the amino acid level, a 


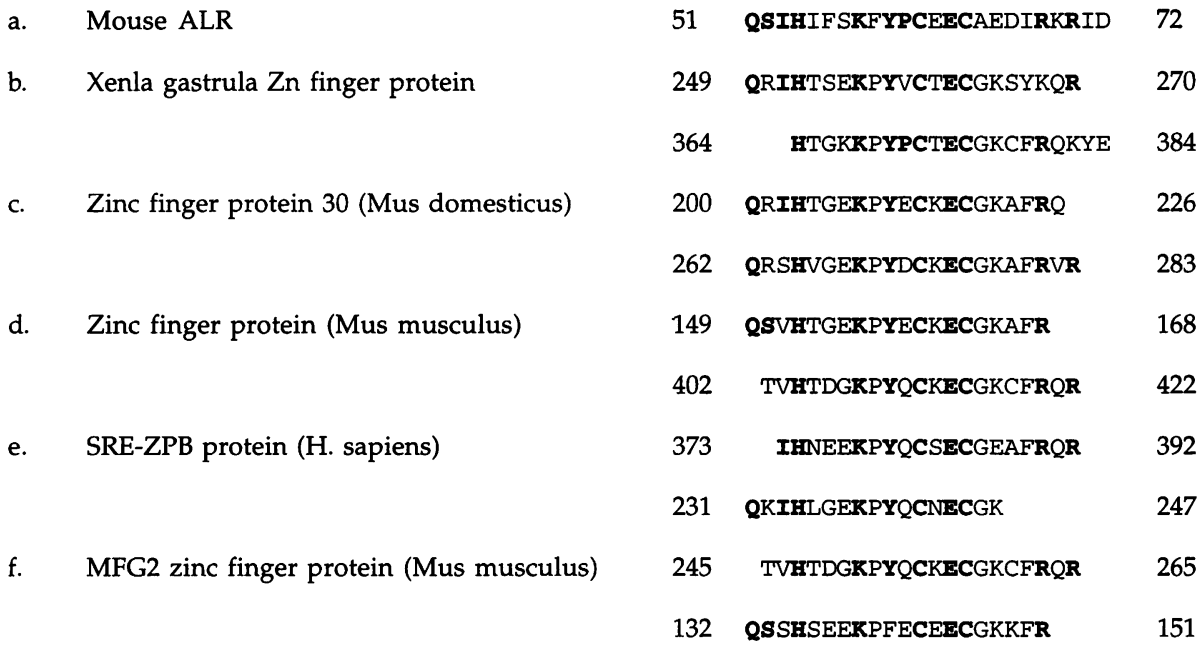

FIG. 7. Similarity of ALR to zinc-finger motifs

Conserved residues are indicated in bold. Accession numbers for the zinc-finger proteins are: b, pir S06579 (27); c, NCBI gi 456269; d, NCBI gi 348994; e, pir S24549; f, M28514 (28). The proteins were aligned with BLAST (29).

conservation that extends to the dual function yeast ERV-1 protein ( $=50 \%$ homology) that is essential for oxidative phosphorylation and mitotic division. This observation, combined with the ubiquitous distribution of ALR, argues for a fundamental role of the protein in some basic process that is operational in all cells. The synthesis of higher amounts of ALR protein in some organs, such as the liver and above all the testis, suggests that this role may be especially relevant to the function of these organs. If, as we have postulated (10), the ALR gene is the mammalian version of the ERV-1 gene and codes for a component involved in the synthesis or stability of nuclear and mitochondrial transcripts, studies of the liver are apposite in the first instance because of this organs' importance in respiratory chain physiology, and in the second because of the liver's unusual capacity for regeneration.

The observation that a portion of the ALR protein has identity with the binding motif in zinc finger proteins (Fig. 7) is both surprising and intriguing. No other protein is known to contain only one-half of a zinc finger motif, and it will be interesting to find out the relation of this finding to ALR function. Transfection studies, and possibly the generation of chimeric knock-out animals, will be needed to test any explanatory hypotheses. If, as suspected, the gene is critical to cell function, the inability to achieve survival with its deletion will be similar to that encountered with hepatocyte growth factor (HGF) (30, 31). Remarkably, HGF is thought to mediate a motogenic, mitogenic, and morphogenetic signal exchange between mesenchyme and epithelia during mammalian development (32). We suspect that the same is true with ALR, and that the family of eight liver augmenters delineated with the Eck fistula model, have a commonality of end points. The regulation of $5^{\prime}$ cap function rate of translation, and thereby cell growth, has been shown for insulin and other growth factors (33).

Finally, it is worthwhile to note that, from the analysis of backcross mice, the ALR gene maps to the $T / t$ region of chromosome 17 of the mouse $(17,18)$. This chromosomal region is marked by a conventional dominant mutant gene, called $T$, and by a series of complicated recessive $t$-mutants that affect large regions of the chromosome. The $t$-alleles are either lethal, or male sterile, when homozygous. In one of the truly bizarre features on this genetic system, male mice of $t /+$ genotype often transmit their mutant $t$-alleles to many more than the expected $50 \%$ of their offspring.

Apparently, spermatozoa carrying a $t$-mutated haplotype interfere with the viability of the spermatozoa carrying the + chromosome, leading to increased transmission of the $t$-allele. The same interaction is presumed to lead to sterility, by mutual spermatozoa destruction, in $t / t$ mice. This interaction implies the occurrence of a chromosome 17-coded, spermatozoa-specific, signaling interaction that is remarkably reminiscent of the signaling role of ALR, and other hepatotropic factors, in hepatocyte survival and proliferation. 
It might also be speculated that this same mechanism of cellular interaction might account for some of the effects of mutant $t$-alleles in embryonic development, thus leading to their lethal phenotypes.

Although a number of candidate $T / t$-region gene products that are expressed in the testes have been identified, none have been able to account completely for these signaling effects of $T / t$-region alleles. In this regard, it is of great interest that we have found ALR mRNA to be strongly expressed in the testes, and immunofluorescence studies have revealed that the ALR protein is located within the germ cells of the mouse testes (Chyung, Kang, Rao, Starzl, and Michaelson, in preparation). Thus, it is clear that ALR must also be considered a candidate gene product that may account for some of the fascinating phenotypic effects of the $T / t$ region, in both embryonic development and sperm function.

\section{ACKNOWLEDGMENTS}

We would like to thank Dr. Abdul Rao, who kindly provided us with the anti-ALR serum; Pete Kang and Yung Chyung for sharing their unpublished immunofluorescence results; Read Fritsch, who electronically composed the figures; and Patrick Hnidka, for typing and editing the manuscript.

\section{REFERENCES}

1. Francavilla A, Hagiya M, Porter KA, Polimeno L, Ihara I, Starzl TE. (1994) Augmenter of liver regeneration (ALR): Its place in the universe of hepatic growth factors. Hepatology 20: 747-757.

2. LaBreque DR, Pesch LA. (1975) Preparation and partial characterization of hepatic regenerative stimulation substance (HSS) from rat liver. J. Physiol. (Lond.) 248: 273-284.

3. Terblanche J, Porter KA, Starzl TE, Moore J, Patzelt L, Hayashida N. (1980) Stimulation of hepatic regeneration after partial hepatectomy by infusion of a cytosol extract from regenerating dog liver. Surg. Gynecol. Obstet. 151: 538-544.

4. McJunkin FA, Breuhaus HC. (1931) Homologous liver as a stimulus to hepatic regeneration. Arch. Pathol. 12: 900-908.

5. Teir H, Ravanti K. (1953) Mitotic activity and growth factors in the liver of the whole rat. Exp. Cell Res. 5: 500-507.

6. Blomqvist K. (1957) Growth stimulation in the liver and tumor development following intraperitoneal injections of liver homogenates in the rat. Acta. Pathol. Microbiol. Scand. 121 (Suppl): 121.

7. LaBrecque DR, Pesch LA. (1975) Preparation and partial characterization of hepatic regenerative stimulator substance (HSS) from rat liver. J. Physiol. 248: 273-284.

8. Francavilla A, Ove $\mathrm{P}$, Polimeno $\mathrm{L}$, et al. (1987) Extraction and partial purification of hepatic stimulatory substance in rats, mice and dogs. Cancer Res. 47: 5600-5605.

9. Francavilla A, Polimeno L, Barone M, Azzarone A, Starzl TE. (1993) Hepatic regeneration and growth factors. J. Surg. Oncol. 3(Suppl): 1-7.

10. Hagiya $M$, Francavilla A, Polimeno L, et al. (1994) Cloning and sequence analysis of the rat augmenter of liver regeneration (ALR) gene: Expression of biologically active recombinant ALR and demonstration of tissue distribution. Proc. Natl. Acad. Sci. U.S.A. 91: 8142-8146.

11. Starzl TE, Jones AF, Terblanche J, Usui S, Porter KA, Mazzoni G. (1979) Growth-stimulating factor in regenerating canine liver. Lancet 1: 127-130.

12. Starzl TE, Watanabe K, Porter KA, Putnam CW. (1976) Effects of insulin, glucagon, and insulin/glucagon infusions on liver morphology and cell division after complete portacaval shunt in dogs. Lancet 1(7964): 821825.

13. Mazzaferro V, Porter KA, Scotti-Foglieni CL, et al. (1990) The hepatotrophic influence of cyclosporine. Surgery 107: 533-539.

14. Starzl TE, Porter KA, Mazzaferro V, Todo S, Fung J, Francavilla A. (1991) Hepatotrophic effects of FK 506 in dogs. Transplantation 51: 67-70.

15. Starzl TE, Schrieber SL, Albers MW, Porter KA, Foglieni CS, Francavilla A. (1991) Hepatotrophic properties in dogs of human FKBP, the binding protein for FK506 and rapamycin. Transplantation 52: 751-753.

16. Lisowski T. (1992) Dual function of a new nuclear gene for oxidative phosphorylation and vegetative growth in yeast. Mol. Gen. Genet. 232: 58-64.

17. Huw LY, Goldsborough AS, Willison K, Artzt K. (1995) Tctex2: A sperm tail surface pro- 
tein mapping to the t-complex. Devel. Biol. 170: $183-194$.

18. Silver LM. (1993) The peculiar journey of a selfish chromosome: Mouse t haplotype and meiotic drive. Trends Genet. 9: 250-254.

19. Maniatis T, Fritsh EF, Sambrook J. (1982) Molecular Cloning: A Laboratory Manual. Cold Spring Harbor Laboratory Press, Cold Spring Harbor, NY.

20. Giorda R, Weisberg EP, Ip TK, Trucco M. (1992) Genomic structure and strain-specific expression of the natural killer cell receptor NKR-P1. J. Immunol. 149: 1957-1963.

21. Devereux J, Haeberli P, Smithies O. (1984) A comprehensive set of sequence analysis programs for the VAX. Nucleic Acids Res. 12: 387.

22. Dignam JD. (1990) Preparation of extracts from higher eucharyotes. Methods Enzymol. 182: 194-202.

23. Sambrook J, Fritsh EF, Maniatis T. (1989) Molecular Cloning: A Laboratory Manual. 2nd Ed. Cold Spring Harbor Laboratory Press, Cold Spring Harbor, NY.

24. Moshier JA, Deutch H, Huang RC. (1987) Structure and in vitro transcription of a mouse $\mathrm{Bl}$ cluster containing a unique $\mathrm{Bl}$ dimer. Gene 58: 19-27.

25. Copeland NG, Gilbert DJ, Jenkins NA, et al. (1993) Genome maps IV 1993. Science 262: 67-82.

26. Hagiya M, Francavilla A, Polimeno L, et al. (1995) Cloning and sequence analysis of the rat augmenter of liver regeneration (ALR) gene: Expression of biologically active recombinant ALR and demonstration of tissue distribution. Proc. Natl. Acad. Sci. U.S.A. 92: 3076. Corrections.

27. Nietfeld W, El-Baradi T, Mentzel $\mathrm{H}$, et al. (1989) Second-order repeats in Xenopus laevis finger proteins. J. Mol. Biol. 208: 639659.

28. Passananti C, Felsani A, Caruso M, Amati P. (1989) Mouse genes coding for "zinc-finger"-containing proteins: Characterization and expression in differentiated calls. Proc. Natl. Acad. Sci. U.S.A. 86: 9417-9421.

29. Altshul SF, Gish W, Miller W, Myers EW, Lippman DJ. (1990) Basic local alignment search tool. J. Mol. Biol. 215: 403-410.

30. Schmidt C, Bladt F, Goedecke S, et al. (1995) Scatter factor/hepatocyte growth factor is essential for liver devlopment. Nature 373: 699-702.

31. Uehara Y, Minowa O, Mori C, et al. (1995) Placental defect and embryonic lethality in mice lacking hepatocyte growth factor/scatter factor. Nature 373: 702-705.

32. Sonnenberg E, Meyer D, Weidner KM, Birchmeier C. (1993) Scatter factor/hepatocyte growth factor and its receptor, and c-met tyrosine kinase, can mediate a signal exchange between mesenchyme and epithelia during mouse development. J Cell Biol. 123: 223-235.

33. Pause A, Belsham GJ, Gingras AC, et al. (1994) Insulin-dependent stimulation of protein synthesis by phosphorylation of a regulator of 5'-cap function. Nature 371: 762-777. 